\title{
Salivary Testosterone Levels Under Psychological Stress and Its Relationship with Rumination and Five Personality Traits in Medical Students
}

\author{
Reza Afrisham ${ }^{1,2}$, Sahar Sadegh-Nejadi ${ }^{1,2}$, Omid SoliemaniFar ${ }^{3}$, Wesam Kooti ${ }^{4}$, Damoon Ashtary-Larky ${ }^{1,5}$, \\ Fatima Alamiri ${ }^{6}$, Mohammad Aberomand ${ }^{5}$, Sedigheh Najjar-Asl ${ }^{7}$, and Ali Khaneh-Keshi ${ }^{7}$ \\ ${ }^{1}$ Student Research Committee, Ahvaz Jundishapur University of Medical Sciences, Ahvaz, Iran \\ ${ }^{2}$ Department of Clinical Biochemistry, Faculty of Medicine, Tehran University of Medical Sciences, Tehran, Iran \\ ${ }^{3}$ Department of Human Sciences, Khuzestan Farhangian University, Ahvaz, Iran \\ ${ }^{4}$ Student Research Committee, Kurdistan University of Medical Sciences, Sanandaj, Iran \\ ${ }^{5}$ Department of Clinical Biochemistry, Faculty of Medicine, Ahvaz Jundishapur University of Medical Sciences, Ahvaz, Iran \\ ${ }^{6}$ Sophmore/Health and Medical Studies, Henry Ford Early College at Henry Ford College, Dearborn, Michigan, USA \\ ${ }^{7}$ Department of Psychology, Faculty of Psychology, Behbahan Branch, Islamic Azad University, Behbahan, Iran
}

Objective The purpose of this study was to evaluate the salivary testosterone levels under psychological stress and its relationship with rumination and five personality traits in medical students.

Methods A total of 58 medical students, who wanted to participate in the final exam, were selected by simple random sampling. Two months before the exam, in the basal conditions, the NEO Inventory short form, and the Emotional Control Questionnaire (ECQ) were completed. Saliva samples were taken from students in both the basal conditions and under exam stress. Salivary testosterone was measured by ELISA. Data was analyzed using multivariate analysis of variance with repeated measures, paired samples t-test, Pearson correlation and stepwise regression analysis.

Results Salivary testosterone level of men showed a significant increase under exam stress $(\mathrm{p}<0.05)$. However, a non-significant although substantial reduction observed in women. A significant correlation was found between extroversion $(r=-0.33)$ and openness to experience $(\mathrm{r}=0.30)$ with salivary testosterone $(\mathrm{p}<0.05)$. Extraversion, aggression control and emotional inhibition predicted $28 \%$ of variance of salivary testosterone under stress.

Conclusion Salivary testosterone reactivity to stress can be determined by sexual differences, personality traits, and emotional control variables which may decrease or increase stress effects on biological responses, especially the salivary testosterone.

Psychiatry Investig 2016;13(6):637-643

Key Words Personality traits, Psychological stress, Rumination, Salivary testosterone.

\section{INTRODUCTION}

Stress is an environmental and psychological stimulus that creates mental or physiological responses and may lead to disease in people. Mild stress can be useful for tasks and cognitive performance, while constant and high stress leads to anxiety

Received: September 30, 2015 Revised: January 4, 2016

Accepted: February 12, 2016 Available online: September 9, 2016

$\triangle$ Correspondence: Mohammad Aberomand, $\mathrm{PhD}$

Department of Clinical Biochemistry, Faculty of Medicine, Ahvaz Jundishapur University of Medical Sciences, Ahvaz 15794-61357, Iran

Tel: +98-9168373935, Fax: +98-6133736832, E-mail: aberumand@yahoo.com

(a) This is an Open Access article distributed under the terms of the Creative Commons Attribution Non-Commercial License (http://creativecommons.org/licenses/by$\mathrm{nc} / 3.0$ ) which permits unrestricted non-commercial use, distribution, and reproduction in any medium, provided the original work is properly cited. and depression. ${ }^{1}$ When stresses occur in the body, sympathetic-adrenal-medullary (SAM) reacts through norepinephrine secretion and Hypothalamic-pituitary-adrenal (HPA) is activated by cortisol secretion. ${ }^{2}$ However, serum, urine and saliva cortisol are classical biomarkers to measure. ${ }^{3,4}$

Testosterone is a steroid hormone that is secreted during 24 hours by the testicles and has powerful anabolic effects on skeletal muscle. ${ }^{5}$ Testosterone level fluctuates during the day, with very high levels in the morning and very low levels in the evening. ${ }^{6}$ The relationship between low testosterone levels and sleep disorders has been demonstrated. ${ }^{7}$ Previous studies have shown that salivary testosterone is reflected in the function of sexual glands and its circadian secretion. Similar to the serum testosterone concentrations, salivary testosterone ex- 
hibits a 24-hour rhythm that is significantly higher during the morning hours than during sunset. ${ }^{8}$ However, some findings ${ }^{9}$ suggest that there was a significant negative relationship between Composite Scale of Morningness (CSM) scores and salivary testosterone level, Sleep duration was uncorrelated with salivary testosterone. similarly, it has been found that a significant negative association exist between the ratio of morning and evening salivary testosterone and age. ${ }^{6}$ Recently, research evidence suggests that testosterone levels are reduced in response to stress. For example, low levels of serum testosterone have been reported during psychological stress, physical stress and actual stress (such as surgery). ${ }^{10}$ moreover, it is recognized that the relation between plasma testosterone levels in men with psychological stress is non-linear (a threshold effect) and direct and linear correlation in women. ${ }^{11}$ Research has also found that anticipatory stress can reduce testosterone levels in men. ${ }^{12}$ Moreover, unlike the men with normal testosterone levels, men with low testosterone levels tend to have more anxiety and irritability. ${ }^{13}$

Academic exams are considered one of the acute psychological stressors, because success in exams is related to the future professional life of students. ${ }^{14}$ Exams (as psychological stress) play an important role in the assessment of learning outcomes and competence of students. In particular, medical students experience a high level of anxiety during exams. Previous studies showed that exam stress can increase anxiety, salivary cortisol level and blood pressure. ${ }^{10}$ However, there are few studies on the relationship between testosterone levels, and the exam psychological stress. A study showed that exam stress increases the anxiety and salivary cortisol levels of male students significantly, but decreased their salivary testosterone level. ${ }^{10}$ While, previous studies have presented valuable neurobiological findings related to men, there is less knowledge in this field for a more general population of both men and women.

As a psychological construct in relation to stress, rumination can lead to the initiation and maintenance of physiological activities related to stress. ${ }^{15}$ The process of rumination is constant and is involuntary thinking about negative events. ${ }^{16}$ Theatrically, rumination is a multifaceted and contains certain aspects of depression, such as weakness of executive control, creating negative thoughts, and insisting on the mental negative status. ${ }^{17}$ Rumination is important in both maintenance and the increase of the severity of depression and stress and its increase is associated with a rise in perceived stress. ${ }^{18}$ To date, research findings confirm the relationship between rumination and responses of cortisol ${ }^{15}$ and salivary alpha amylase ${ }^{19}$ to stress. However, empirical research lacking on the relationship between salivary testosterone and the trait of rumination, as a psychological characteristics associated with stress. For this reason, we have tried to study the relationship between rumination and salivary testosterone under psychological stress.

Currently, literature states that people display different physiological reactions significantly in dealing with a challenging environment. In relation to the HPA system, consistent individual differences in reactivity to stress have been observed using cortisol, however in the SAM system, it was related to cardiovascular activity. What is less discussed is whether the individual differences in biological reactivity to stress are, reflecting differences in basic human personality traits or not. There is evidence that shows certain components of type A behavior, such as hostility, is associated with more cortisol and cardiovascular responses to stress. ${ }^{20}$

Several previous studies have attempted to explore the possible relationship between stress and personality variables, such as hardiness, sensation seeking, achievement motivation, extraversion and neuroticism, however, these studies have been conducted using higher levels of personality dimension measurement tools that contain both specific groups and have many internal correlations. ${ }^{18}$ In particular, testosterone level is related to a number of behavioral and psychosocial variables, such as feelings of hostility, anger, impulsivity, and aggression. ${ }^{11}$

It appears that personality can be better explained by the Five Factor Model of personality, which offers a normal structure of personality traits using the Personality Inventory NEOPI. This model has been widely accepted as the most prominent structure of personality. According to this model, the interpersonal variance in personality traits is explained through five major dimensions of personality, such as extraversion, agreeableness, conscientiousness, neuroticism, and openness to experience. ${ }^{21}$ Using this model, previous studies have reported a negative correlation between neuroticism with cortisol $^{22}$ and salivary alpha-amylase $\mathrm{e}^{19,23}$ as well as the finding of a negative $^{23}$ and positive ${ }^{19}$ correlation between agreeableness with salivary alpha-amylase. Nevertheless, our knowledge about the relationship between the five personality traits and salivary testosterone under psychological stress is still low. In fact, there is a possibility that the salivary testosterone levels under stressful conditions can be predicted by personality traits. Therefore, in this study, we examined the relationship between rumination and personality traits with salivary testosterone under psychological stress in medical students.

\section{METHODS}

\section{Participants}

In this cross-sectional descriptive study, the population included all the government medical students at Ahvaz University of Medical Sciences who wanted to participate in a final exam. The inclusion criteria for the study included the ab- 
sence of infectious disease; autoimmune; hereditary immune deficiency; mental illness; lack of drugs to suppress the immune system (immunosuppressive); no vaccine or blood; endocrine diseases such as diabetes; non-smoking; and lack of surgery during two months before saliva sampling. Using Cochran formula and a simple random sampling method, 58 students (28 female; 30 male) were selected. Due to the students being freshmen, the first exam was considered as a stressful situation. The natural condition did not significantly change the normal levels of biochemical factors, but in stress condition or mental pressure, changes are obvious. Thus, the exam was considered a psychological stressful situation. ${ }^{19,24}$ Data was collected using the personality Neo-short form (FFI-NEO) and Emotional Control Questionnaire (ECQ).

\section{Questionnaires}

\section{The personality Neo-short form}

In this study, McCrae and Costa's 60-items questionnaire (1992) was used. This scale measures the five major personality traits, which include: Neuroticism (N), Extraversion (E), Agreeableness (A), Openness to Experience (O), and conscientiousness (C). Each of these traits can be measured with 12 questions. The answer to the questionnaire is set based on a five-point Likert scale: completely disagree (0); disagree (1); indifferent (2); agree (3); and completely agree (4). Based on Cronbach's alpha coefficients by Costa and McCrae, the internal consistency of this scale for traits of $\mathrm{N}, \mathrm{E}, \mathrm{O}, \mathrm{A}$, and $\mathrm{C}$ was $0.66,0.77,0.73,0.68$, and 0.81 , respectively. ${ }^{25}$ In this study, using Cronbach's alpha, the coefficient of reliability of the questionnaire calculated for the traits of $\mathrm{N}, \mathrm{E}, \mathrm{O}, \mathrm{A}$, and $\mathrm{C}$ was $0.76,0.68,0.77,0.72$, and 0.81 , respectively.

\section{Emotional Control Questionnaire}

The questionnaire was developed by Roger and Nesshoever (1987) and was renewed by Roger and Najarian (1989). The ECQ measures four scales: Emotional Inhibition, aggression control, ruminants or rehearsing and benign control measures; it has 56 items. Each sub-scale has 14 questions. The score of a person in each sub-scale ranges from zero to 14 and in the total scale ranges from zero to 56 . High scores indicate greater emotional control. ${ }^{26}$ The validity of the questionnaire, using internal consistency, and Cronbach's alpha coefficient calculated by Rafineya et al., ${ }^{27}$ for each sub-scale of emotional inhibition, aggression control, rumination, and benign control were $0.70,0.76,0.77$, and 0.58 , respectively and its total validity was 0.68 . In the study, the reliability coefficient obtained using Cronbach's alpha coefficient for the total scale was 0.78 and for each of the sub-scales of emotional inhibition, aggression control, rumination, and benign control was 0.66 ,
$0.63,0.68$ and 0.73 , respectively.

\section{Methods}

To conduct the research in the first phase, two months before the exam (basal condition), all participants completed the Personality NEO- short form questionnaire and emotional control Questionnaires (ECQ). Then, two days later, saliva samples were taken to measure salivary testosterone in the basal conditions. In the second phase, once again to measure salivary testosterone during exam stress, saliva samples were taken at the start of the exam. In both phases of sampling, all participants were asked not to have caffeine of the night before taking the saliva samples until the taking of the samples, and were not permitted to eat any food one hour prior to the exam. ${ }^{19,26}$ In order to complete the saliva sampling, subjects were asked to collect their saliva for five minutes in tubes determined by passive methods. ${ }^{19,28}$ Sampling was performed in the same circumstances and particular time (10 am). Then, samples were centrifuged in $3000 \mathrm{~g}$ for $15 \mathrm{~min}$ and the supernatant stored at $-70^{\circ} \mathrm{C}$. testosterone level was measured by ELISA method (Diametra, Italy).

\section{Ethical principles}

This project was approved by the code of ethics of ajums. REC.1393.442 in student research committee of Ahvaz Jundishapur University of Medical Sciences.

\section{Statistical analyses}

Statistical analysis was performed with SPSS version 18 . Data was analyzed using descriptive statistical methods including mean and standard deviation, and multivariate analysis of variance with repeated measures for the effects of within-subjects factor (salivary testosterone levels measured at 2 steps) and interaction with gender, paired samples t-test to compare the difference in salivary testosterone of the basal conditions and under exam stress, and the Pearson correlation coefficient and stepwise regression analysis.

\section{RESULTS}

\section{Demographic and Clinical Characteristics}

The summary statistics concerning the demographic characteristics and questionnaires data for all study participants $(\mathrm{n}=58)$ are presented in Table 1 . The study participants ranged in age from 19-22 years, with a mean age of $19.5 \pm 0.712$ years. There were 30 male participants (51.7\%) and 28 female participants (48.27\%). Mean and standard deviation for the neuroticism, extraversion, openness to experience, agreeableness, conscientiousness, and rumination were $22.56 \pm 4.8$, $25.4 \pm 5.08,25.54 \pm 4.24,27.11 \pm 4.0,31.04 \pm 5.58$ and $7.63 \pm 2.28$, 
respectively (Table 1 ).

\section{Multivariate analysis of variance with repeated measures for within-subjects factor and interaction effects}

Mauchly's Test's results with $\mathrm{p}=0.037$ rejected an assumption of normal distribution. For this reason, we used the Greenhouse-Geisser correction test for the testing of the effect of within-subjects factor (salivary testosterone levels measured at 2 steps) and interaction effects between within-subjects factor and gender. Box's $M$ test results $(p=0.014,21.06)$ about equality of variance matrix showed that the assumption of homogeneity of variance-covariance matrix is established. According to the results of the multivariate analysis of variance with repeated measures (Table 2), significant difference was not observed between salivary testosterone levels measured at two steps (within-subjects factor). However, interaction be-

Table 1. Demographic and clinical characteristics of the study subjects $(\mathrm{N}=58)$

\begin{tabular}{lc}
\hline \multicolumn{1}{c}{ Variable } & Mean $\pm \mathrm{SD}(\mathrm{N}, \%)$ \\
\hline Age & $19.5 \pm 0.712$ \\
Sex & \\
Male & $30(51.7 \%)$ \\
Female & $28(48.27 \%)$ \\
Rumination & $7.63 \pm 2.28$ \\
Five personality traits & \\
Neuroticism & $22.56 \pm 4.8$ \\
Extraversion & $25.4 \pm 5.08$ \\
Openness & $25.54 \pm 4.24$ \\
Agreeableness & $27.11 \pm 4.0$ \\
Consciousness & $31.04 \pm 5.58$ \\
\hline
\end{tabular}

tween within-subjects factor and gender was significant $(\mathrm{p}=$ 0.003) (Table 2).

\section{Comparison of salivary testosterone levels in the basal conditions and under exam stress according to gender}

The difference of salivary testosterone levels in the basal conditions and under exam stress, according to gender of the subjects, was obtained using paired samples t-test (Table 3). According to results displayed in Table 3, male students' salivary testosterone levels under exam stress showed a significant increase compared to the basal conditions $(p=0.05)$. A statistically significant difference was not found in mean scores of female students' salivary testosterone and even within the entire sample (Table 3).

\section{Correlation of five personality traits and rumination with salivary testosterone}

According to the results in Table 4, only traits of extraversion $(\mathrm{p}<0.05, \mathrm{r}=-0.33)$ and openness to experience $(\mathrm{p}<0.05$, $r=0.30$ ) were significantly correlated with salivary testosterone under exam stress. However, in the basal conditions, only a positive correlation existed between openness to experience and salivary testosterone $(\mathrm{p}<0.01, \mathrm{r}=0.43)$. Significant correlation was not observed for rumination and other personality traits (Table 4).

\section{Stepwise regression analysis}

In order to predict Salivary testosterone on the exam condition, a Stepwise regression analysis was used (Table 5). The results of the regression analysis indicated that only the trait of Extraversion and subscales of emotional inhibition and of aggression control from the Emotional Control Questionnaire

Table 2. Results of multivariate analysis of variance with repeated measures for within-subjects factor and interaction effects ( $N=58)$

\begin{tabular}{lcccccccc}
\hline \multicolumn{1}{c}{ Source of Effects } & Test & SS & DF & MS & F & Sig & ES & SP \\
\hline W-SF & Greenhouse-Geisser & 15449.5 & 1 & 15449.5 & 2.76 & 0.104 & 0.062 & 0.369 \\
Interaction of W-SF and gender & Greenhouse-Geisser & 55000.0 & 1 & 55000.0 & 9.85 & 0.003 & 0.190 & 0.866
\end{tabular}

SS: sum squares, MS: mean square, ES: eta square, SP: statistical power, W-SF: within-subjects factor [salivary testosterone levels (pg/mL) measured at two steps]

Table 3. Comparison the mean salivary testosterone $(\mathrm{pg} / \mathrm{mL})$ in basal conditions and under exam stress in the total sample and according to gender

\begin{tabular}{|c|c|c|c|c|c|c|c|}
\hline \multirow{2}{*}{ Groups } & \multicolumn{2}{|c|}{ BST } & \multicolumn{2}{|c|}{ STE } & \multirow{2}{*}{ df } & \multirow{2}{*}{$\mathrm{t}$} & \multirow{2}{*}{$\mathrm{p}$} \\
\hline & Mean & $\mathrm{SD}$ & Mean & SD & & & \\
\hline Male $(\mathrm{N}=30)$ & 149.45 & 78.54 & 225.95 & 155.27 & 29 & -2.63 & $0.016^{*}$ \\
\hline Fenale $(\mathrm{N}=28)$ & 105.27 & 45.36 & 89.40 & 88.60 & 27 & 0.890 & 0.383 \\
\hline Total $(\mathrm{N}=58)$ & 127.36 & 67.21 & 157.68 & 142.75 & 57 & -1.66 & 0.104 \\
\hline
\end{tabular}

${ }^{*} \mathrm{p}<0.05$. BST: basal salivary testosterone, STE: salivary testosterone under exam 
with coefficients ( $\beta$ ); $-0.33,0.33$ and -0.27 respectively, were able to predict $28 \%$ of salivary testosterone on the exam condition (Table 5).

\section{DISCUSSION}

The purpose of this study was to evaluate the salivary testosterone under psychological stress and its relationship with rumination and five personality traits in medical students. As shown in Table 1, the interaction between the internal factor (salivary testosterone levels in 2 steps) and gender was significant. Indeed, psychological stress cannot alone make a difference of salivary testosterone levels in two stages, but gender differences interfere in salivary testosterone reactivity to stress. In addition, in male students, salivary testosterone level under psychological stress was significantly higher than in the two months before exam (the basal conditions), while, the difference was not significant in female students, However, salivary testosterone level under psychological stress was lower than in the two months before exam (the basal condition). Still, in the total sample, testosterone level increased during exam stress, which was non-significant. Based on these findings, it is possible that salivary testosterone levels in stressful

Table 4. Correlation of FPT, rumination with salivary testosterone level $(\mathrm{pg} / \mathrm{mL})$ in basal and exam situations $(\mathrm{N}=58)$

\begin{tabular}{lcc}
\hline \multirow{2}{*}{ Variable } & BST & STE \\
\cline { 2 - 3 } & Pearson's $\mathrm{r}$ & Pearson's $\mathrm{r}$ \\
\hline FPT & & \\
Neuroticism & 0.039 & 0.085 \\
Extraversion & -0.27 & $-0.33^{*}$ \\
Openness & $0.43^{* *}$ & $0.30^{*}$ \\
Agreeableness & -0.025 & -0.15 \\
Consciousness & 0.16 & 0.19 \\
ECQ & & \\
Rumination & 0.043 & 0.019 \\
Emotional inhibition & -0.18 & -0.27 \\
Aggression control & 0.11 & 0.20 \\
Benign control & -0.060 & 0.020 \\
\hline *p $<0.05, * * p<0.001$. BST: basal salivary testosterone, STE: salivary \\
testosterone under exam, FPT: five personality traits, ECQ: Emo- \\
tional Control Questionnaire
\end{tabular}

conditions may act as a stress response related to gender. In other words, psychological stressors depend on gender and this possibly leads to different biological responses, such as increased salivary testosterone level in men or lower level in women. This dichotomy may be more effective in stress reactivity. In fact, the gender differences can be an inseparable part of testosterone reactivity to stress. ${ }^{29}$ Previous study, reported a significant increase in male students' salivary testosterone in response to psychosocial stressors. ${ }^{30}$ In another study on firstyear medical students, in order to assess the stress-reducing effects of MBMS (Mind Body Medicine Skills Program), changes in levels of cortisol, DHEA-S, testosterone, and IgA were measured in both groups [(T2p)-post-intervention and (T2c)-control] during the period of final examinations. The results, after implementation of the intervention program (MBMS) showed that cortisol, DHEA-S, and testosterone levels at T2p were significantly lower than T2. ${ }^{31}$ Also, experimental evidence on young animals, such as pigs, shows that psychological stress (social stimulation) resulted in a significant increase in testosterone levels and cortisol reduction in young male pigs; whereas these changes were not observed in the female pigs. ${ }^{32}$ However, there are findings that indicate lower levels of plasma testosterone in response to psychological and physical stress, actual stress (surgery), ${ }^{33}$ anticipatory stress, ${ }^{12}$ and in particular, the reduction of salivary testosterone in exam stress objective structured clinical examination (OSCE). ${ }^{10}$ This inconsistency of results may be due to differences in the stressful situation and the possible consequences associated with those conditions. For example, previous studies on humans $^{34,35}$ and animals ${ }^{36,37}$ indicate that in competitive sports or fights (as a stressful situation), winning or losing can respectively increase or decrease testosterone levels. Also, these inconsistencies may be the reason that relationship of testosterone reactivity and stress follows a U-shaped pattern; namely, a non-linear correlation (a threshold effect) for men and a direct and linear pattern for women. Based on the pattern, lower levels of stress can lead to increased levels of testosterone in men up to a certain threshold, which is characterized by a positive association, while reduced levels of testosterone can be observed by high levels of stress, with a subsequent leveling off. Therefore, the relationship between stress and testosterone reactivity is determined by a negative associa-

Table 5. Stepwise regression analysis to predict salivary testosterone $(\mathrm{pg} / \mathrm{mL})$ under exam condition $(\mathrm{N}=58)$

\begin{tabular}{ccccccc}
\hline Model & Variables & MR & $\mathrm{R}^{2}$ & $\mathrm{~F}$ & $\mathrm{~b}$ & $\mathrm{p}$ \\
\hline 1 & Extraversion & 0.333 & 0.11 & 5.23 & -0.33 & 0.027 \\
2 & AC & 0.463 & 0.21 & 5.60 & 0.339 & 0.025 \\
3 & EI & 0.537 & 0.28 & 5.39 & -0.27 & 0.049 \\
\hline
\end{tabular}

AC: aggression control, EI: emotional inhibition 
tion in higher levels of stress. ${ }^{11}$ Nonetheless, the complex relationship between testosterone and stress requires more research in the field of psych-neuro-endocrinology.

Another hypothesis of this study was that salivary testosterone levels under stressful conditions were anticipated by rumination and five personality traits. Unlike what we expected, rumination with salivary testosterone was unrelated. In contrast, as mentioned above, previous studies have found a relationship between rumination with salivary cortisol ${ }^{15}$ and amylase salivary. ${ }^{19}$ Our findings suggest that the traits of extraversion and openness to experience have respectively a negative and positive correlation with salivary testosterone levels under stress. These associations suggest that the trait of extraversion in both genders can be associated with reduced salivary testosterone. Comparing these associations with the basal conditions indicates that there is a relatively constant pattern of correlation with a slight difference between extraversion and openness to experience with salivary testosterone in both situations.

Personality traits can be effective on a person's perception of the stressful situation and this allows for different biological reactions. Cognitive stress theory and previous research have shown that personality influences the biological responses to stress. ${ }^{20} \mathrm{~A}$ remarkable finding of the present study was that the results of regression analysis showed that the traits of extraversion, emotional inhibition, and aggression control have more roles in predicting of the salivary testosterone under psychological stress. According to Beta coefficients obtained, extraversion and emotional inhibition can predict a reduction of salivary testosterone under psychological stress, while aggression control predicts higher level of salivary testosterone under stress. Previous studies emphasize the role of hormones, especially testosterone and cortisol, as risk factors for aggression and violence..$^{38}$ However, it is suspected that anger and aggression are influenced by mechanisms of emotional control and executive. Also, with regard to aggression, self-regulation, and regulation of emotions, the perspectives of psychology, psychiatry, and neuroscience insists on the role of the control mechanism of the prefrontal cortex on anger and aggression. Recently, researchers have suggested an increase in functional connectivity between the amygdala and a network of top-down controls of the prefrontal cortex during control of anger. This connection is stronger in people with high testosterone ${ }^{39}$ In general, emotional inhibition and aggression control through the activation of neurochemical mechanisms in the brain, particularly as previous studies have shown that the prefrontal cortex, may increase or decrease the testosterone reactivity to stress. This can be the basis for individual differences in biological reactivity to stress. ${ }^{39}$ It may also be the main reason there are individual differences in biological re- activity to stress.

In summary, our findings indicate that the five-factor personality model and variables associated with emotional control (ECQ) may explain individual differences in salivary testosterone reactivity to psychological stress. In fact, as mediating variables, they increase or reduce the effects of stress. However, minimal studies exist about the relationship between psychological characteristics, especially five personality traits, with salivary testosterone under psychological stress. So, this study could be considered as a controversial and new study, but many of the psychological variables are mutable, and unlike other experimental variables, are less stable. Therefore, we need to perform further studies on psychological and personality factors and their interaction with gender under stress condition. Also, with regard to the relationship between sleep ${ }^{7,9}$ and circadian cycles $^{5,6,8}$ with testosterone levels, we propose that in future studies, interaction between personality traits and testosterone based on these two variables be evaluated, for the reason that the circadian rhythm of testosterone, According to various personality dimensions is also different.

In conclusion, in this study, we observe that salivary testosterone reactivity to psychological stress is different in both genders. Men's response to stress leads to high level of salivary testosterone, but women's response to stress shows reduction in salivary testosterone. In addition, we found that personality characteristics and emotional control variables are associated with biological responses to stress, particularly salivary testosterone.

\section{Acknowledgments}

This article has been adapted from a research project approved by the code of 92 s86 and ethics code ajums. REC. 1393.442 in the Student Research Committee University of Ahvaz Jundishapur University of medical sciences; we appreciate the student research committee for preparation of grant. In addition, authors thank all medical students who participated in this study.

\section{REFERENCES}

1. Joshi RM, Sanghavi SJ, Upadhyaya DP, Chauhan A, Halvadia S. Effect of examination stress on the plasma cortisol level. Nat J Med Res 2012; 2:435-438.

2. Ohura K, Nozaki T, Shinohara M, Daito K, Sonomoto M, Daito M. Utility of salivary biomarker for stress induced by dental treatment. Jpn Dent Sci Rev 2012;48:14-17.

3. Kim SB, Takenaka Y, Torimura M. A bioluminescent probe for salivary cortisol. Bioconjug Chem 2011;22:1835-1841.

4. Levine A, Zagoory-Sharon O, Feldman R, Lewis JG, Weller A. Measuring cortisol in human psychobiological studies. Physiol Behav 2007;90:43-53.

5. Spratt DI, O’Dea LS, Schoenfeld D, Butler J, Rao PN, Crowley WF Jr. Neuroendocrine-gonadal axis in men: frequent sampling of $\mathrm{LH}, \mathrm{FSH}$, and testosterone. Am J Physiol 1988;254:E658-E666.

6. Bribiescas RG, Hill KR. Circadian variation in salivary testosterone across age classes in Ache Amerindian males of Paraguay. Am J Hum Biol 2010;22:216-220. 
7. Wittert G. The relationship between sleep disorders and testosterone in men. Asian J Androl 2014;16:262-265.

8. Kraemer WJ, Loebel CC, Volek JS, Ratamess NA, Newton RU, Wickham RB, et al. The effect of heavy resistance exercise on the circadian rhythm of salivary testosterone in men. Eur J Appl Physiol 2001;84:1318.

9. Randler C, Ebenhöh N, Fischer A, Höchel S, Schroff C, Stoll JC, et al. Chronotype but not sleep length is related to salivary testosterone in young adult men. Psychoneuroendocrinology 2012;37:1740-1744.

10. Choi J, Chung M, Lee Y. Modulation of pain sensation by stress-related testosterone and cortisol. Anaesthesia 2012;67:1146-1151.

11. King JA, Rosal MC, Ma Y, Reed GW. Association of stress, hostility and plasma testosterone levels. Neuro Endocrinol Lett 2005;26:355-360.

12. Schulz P, Walker JP, Peyrin L, Soulier V, Curtin F, Steimer T. Lower sex hormones in men during anticipatory stress. Neuroreport 1996;7:31013104.

13. Sternbach H. Age-associated testosterone decline in men: clinical issues for psychiatry. Am J Psychiatry 1998;155:1310-1318.

14. Singh R, Goyal M, Tiwari S, Ghildiyal A, Nattu SM, Das S. Effect of examination stress on mood, performance and cortisol levels in medical students. Indian J Physiol Pharmacol 2012;56:48-55.

15. Zoccola PM, Dickerson SS. Assessing the relationship between rumination and cortisol: a review. J Psychosom Res 2012;73:1-9.

16. Smith JM, Alloy LB. A roadmap to rumination: a review of the definition, assessment, and conceptualization of this multifaceted construct. Clin Psychol Rev 2009;29:116-128.

17. van Vugt MK, Hitchcock P, Shahar B, Britton W. The effects of mindfulness-based cognitive therapy on affective memory recall dynamics in depression: a mechanistic model of rumination. Front Hum Neurosci 2012;6:257.

18. Hu E, Koucky EM, Brown WJ, Bruce SE, Sheline YI. The role of rumination in elevating perceived stress in posttraumatic stress disorder. J Interpers Violence 2013;29:1953-1962.

19. Afrisham R, Sadegh-Nejadi S, SoliemaniFar O, Abromand M, Kooti W, Najjar Asl S, et al. Evaluating the salivary alpha-amylase level under psychological stress and its relationship with rumination and the five personality traits. J Mazandaran Univ Med Sci 2015;25:22-33.

20. Bibbey A, Carroll D, Roseboom TJ, Phillips AC, de Rooij SR. Personality and physiological reactions to acute psychological stress. Int J Psychophysiol 2013;90:28-36.

21. Costa PT, McCrae RR. The revised neo personality inventory (neo-pir). The SAGE handbook of personality theory and assessment 2008;2: 179-198.

22. Nater UM, Hoppmann C, Klumb PL. Neuroticism and conscientiousness are associated with cortisol diurnal profiles in adults-role of positive and negative affect. Psychoneuroendocrinology 2010;35:1573-1577.

23. Inukai K, Shinada M, Tanida S, Takahashi C, Mifune N, Takagishi H, et al. Salivary alpha-amylase levels and big five personality factors in adults. Neuro Endocrinol Lett 2009;31:771-774.

24. Ghafourian-Boroujerdnia M SZ, Hamid N, Hemmati AA, Kooti W. The relationship of hardiness and immune system cells. J Isfahan Med Sch 2014;31:1812-1820.

25. Costa PT, McCrae RR. Reply to eysenck. Pers Individ Dif 1992;13:861865.

26. Roger D, Najarian B. The relationship between emotional rumination and cortisol secretion under stress. Pers Individ Dif1998;24:531-538.

27. Rafineya P RTSK AFP. The relation between emotional expression and mental health in students. J Psychol 2006;10:85-104.

28. Afrisham R, Aberomand M, SoliemaniFar O, Kooti W, Ashtary-Larky D, Alamiri F, et al. Levels of salivary immunoglobulin A under psychological stress and its relationship with rumination and five personality traits in medical students. Eur J Psychiatry 2016;30:41-53.

29. Gaudet BM, Katharina S, Jenny PH, Jeremy P, Amanda P, Elizabeth $\mathrm{SH}$. Gender on testosterone-stress reactivity to the trier social stress test. Innovate UNO. Paper 5. March 2013.

30. Clow A, Patel S, Najafi M, Evans PD, Hucklebridge F. The cortisol response to psychological challenge is preceded by a transient rise in endogenous inhibitor of monoamine oxidase. Life Sci 1997;61:567-575.

31. MacLaughlin BW, Wang D, Noone AM, Liu N, Harazduk N, Lumpkin $\mathrm{M}$, et al. Stress biomarkers in medical students participating in a mind body medicine skills program. Evid Based Complement Alternat Med 2011;2011:950461.

32. Lürzel S, Kaiser S, Sachser N. Social interaction, testosterone, and stress responsiveness during adolescence. Physiol Behav 2010;99:40-46.

33. Van den Berghe G. The neuroendocrine response to stress is a dynamic process. Best Pract Res Clin Endocrinol Metab 2001;15:405-419.

34. Gonzalez-Bono E, Salvador A, Serrano MA, Ricarte J. Testosterone, cortisol, and mood in a sports team competition. Horm Behav 1999;35: 55-62.

35. Neave N, Wolfson S. Testosterone, territoriality, and the 'home advantage'. Physiol Behav 2003;78:269-275.

36. Rose RM, Holaday JW, Bernstein IS. Plasma testosterone, dominance rank and aggressive behaviour in male rhesus monkeys. Nature 1971; 231:366-368.

37. van de Poll NE, Smeets J, van Oyen H, van der Zwan S. Behavioral consequences of agonistic experience in rats: sex differences and the effects of testosterone. J Comp Physiol Psychol 1982;96:893-903.

38. Terburg D, Morgan B, Van Honk J. The testosterone-cortisol ratio: a hormonal marker for proneness to social aggression. Int J Law Psychiatry 2009;32:216-223.

39. Denson TF, Ronay R, von Hippel W, Schira MM. Endogenous testosterone and cortisol modulate neural responses during induced anger control. Soc Neurosci 2013;8:165-177. 